\title{
Is the Emergency Cesarean Section associated with Hypotension? Retrospective Analysis of 80 Patients Undergoing Elective or Emergency Cesarean Section under Spinal Anesthesia
}

\author{
Esra Adıyeke
}

Department of Anesthesiology and Reanimation, Sancaktepe Sehit Prof. Dr. Ilhan Varank Training and Research Hospital, Istanbul, Turkey

\begin{abstract}
Introduction: While CS is associated with several intraoperative and postoperative complications, the risk of the hemodynamic changes in case of an emergency CS is usually underestimated. The lack of adequate patient preparation before anesthesia and insufficient time to correct the volume depletion before anesthesia are common issues in emergency CS. The present study aimed to compare the hemodynamic changes in patients undergoing elective or emergency CS.

Methods: All consecutive patients undergoing CS with spinal anesthesia in a tertiary stage hospital were enrolled in this retrospective study. Patients were divided into two groups according to the emergency of the CS as Elective CS group and emergency CS groups. Elective CS patients received routine volume replacement before surgery, whereas emergency CS patients received volume replacement according to the operator's decision. The difference in the hemodynamic parameters throughout the surgery between the elective and emergency CS groups was the primary outcome measure of this study. Results: The amount of the intravenous fluid administered before surgery was significantly lower in the emergency CS group compared to the elective CS group $(650 \pm 280 \mathrm{ml}$ vs. $1430 \pm 460 \mathrm{ml}, \mathrm{p}<0.01)$. A significant drop occurred in systolic, diastolic, and mean arterial pressure early at the 5 th minute of anesthesia in patients undergoing emergency CS. However, no significant change was observed in the elective CS group concerning the systolic, diastolic, and mean arterial pressure and heart rate.

Discussion and Conclusion: In contrast to the women undergoing elective CS, a significant drop occurs in systolic, diastolic and mean arterial blood pressure following the implementation of the spinal anesthesia in patients undergoing emergency CS. Rapid evaluation of the volume status and intravenous volume replacement may prevent the reduction of the arterial pressure in patients undergoing emergency CS.

Keywords: Cesarean section; elective; emergency; hypotension; spinal anesthesia.
\end{abstract}

C esarean section (CS) is among the most common operations in obstetrics with a rising incidence throughout the world. Delayed childbearing, safer anesthesia, and medical litigation are the main causes of this new trend of delivery ${ }^{[1]}$. The rate of CS in Europe is approximately $25 \%$ of all deliveries and is estimated at $32.2 \%$ in the USA ${ }^{[2]}$. The risk of fetal trauma during the vaginal delivery, higher newborn hospitalization rates and higher incidence of the urinary incontinence in the elderly who had undergone vaginal delivery also influence the women's choice favoring $\mathrm{CS}^{[3]}$. Moreover, financial, social, and cultural elements are likely to play an important role in the increasing frequency of the CS.

Correspondence (iletişim): Esra Adıyeke, M.D. Sancaktepe Sehit Prof.Dr. Ilhan Varank Egitim ve Arastirma Hastanesi Anestezi ve Reanimasyon Anabilim Dali, Istanbul, Turkey

Phone (Telefon): +90 5074851869 E-mail (E-posta): dresradiyeke@gmail.com

Submitted Date (Başvuru Tarihi): 19.09.2019 Accepted Date (Kabul Tarihi): 18.10.2019

Copyright 2019 Haydarpaşa Numune Medical Journal

OPEN ACCESS This is an open access article under the CC BY-NC license (http://creativecommons.org/licenses/by-nc/4.0/) 
Malpresentation, cephalopelvic disproportion, multiple pregnancies, severe hypertension, fetal macrosomia, fetal distress, low birth weight, failed induction of labor, placenta previa, placental abruption, maternal pelvic defor-

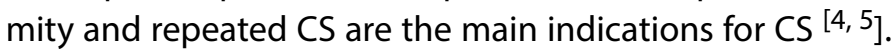
In some instances, emergency CS is indicated if there is an immediate threat to the life of the woman or fetus, such as fetal distress in the first stage, cord prolapse, antepartum hemorrhage, and uterine rupture ${ }^{[6]}$. However, elective CS is also common as a result of the mothers' request or in the presence of increased maternal age, obesity, diabetes mellitus, fertility treatment and relative indications for CS, including pathological cardiotocography, failure to progress in labor, and previous CS.

While CS is associated with several intraoperative and postoperative complications, hemodynamic derangements are frequently encountered, particularly when spinal anesthesia is implemented. The lack of adequate patient preparation before anesthesia and insufficient time to correct the volume depletion prior to anesthesia are common issues in emergency CS, which may further lead to hemodynamic alterations during CS and eventually lead to fetal hypoxia. However, data comparing the hemodynamic alterations in elective and emergency CS are limited. The present retrospective study, therefore, aimed to compare the hemodynamic changes in patients undergoing elective or emergency CS.

\section{Materials and Methods}

All consecutive patients undergoing CS in a tertiary stage hospital between November 2018 and May 2019 were enrolled in this retrospective analysis. Women were eligible if they were having a cesarean section of a singleton fetus greater than 37 weeks of gestation under spinal anesthesia with an American Society of Anesthesiologists Classification (ASA) class below III. Women with preeclampsia, eclampsia, preterm labor, kidney disease, hypertension, and women receiving antihypertensive agents were excluded from the study protocol. Data concerning the demographic characteristics, physical examination, and Intraoperative hemodynamic monitorization were retrieved from the institutional digital database and from the patient charts. Written informed consent was obtained from all subjects included in this study. This study was approved by the Institutional Ethical Committee and was performed in accordance with the recent version of the Helsinki Declaration. Patients were divided into two groups according to the emergency of the CS as Elective CS group and emergency CS groups. Patients in the elective CS group were stopped for oral intake at least 12 hours before the CS. These patients were also administered $1000 \mathrm{ml}$ of saline and $500 \mathrm{ml}$ of lactated Ringer's solution four hours before the surgery. The patients in the emergency CS group were also administered iv. saline, according to the gynecologists' discretion and the amount of the volume administered before the surgery was recorded for each patient.

\section{Anesthesia and Hemodynamic Measurement}

All CS procedures were carried by the same surgical team and the same anesthetist. A standard spinal anesthesia protocol was applied to all participants. Spinal anesthesia was performed with a spinal needle inserted through the L3-L4 interspace. Following the return of clear cerebrospinal fluid, $0.5 \%$ bupivacaine was injected over 20-30 $s$ through a spinal needle in appropriate dose for each patient. Monitoring with pulse oximetry, noninvasive blood pressure measurement and electrocardiogram was established. Systolic arterial pressure, diastolic arterial pressure, mean arterial pressure, heart rate, and oxygen saturation were recorded at six different time points: Before induction (pre-anesthesia), at $5^{\text {th }}, 15^{\text {th }}, 30^{\text {th }}, 40^{\text {th }}$ minute of anesthesia and five minutes after the anesthesia (post-anesthesia). Intravenous ephedrine 5-10 mg was administered provided the systolic blood pressure drops below $90 \mathrm{mmHg}$. Heart rate below 50bats/min was treated with intravenous atropine $1 \mathrm{mg}$.

\section{Primary Outcome}

The elective and emergency CS groups were compared concerning demographic characteristics and the hemodynamic measurements. The difference in the hemodynamic parameters throughout the surgery between the elective and emergency CS groups was the primary outcome measure of this study.

\section{Statistical Analysis}

Statistical analyses were performed using IBM SPSS 20.0 (SPSS Inc., Armonk, NY, USA) software. Continuous variables were expressed as mean \pm standard deviation and categorical variables as number and percentage. The Shapiro-Wilk test was used to assess the distribution of the data. Friedman test with Wilcoxon signed-rank test was used to compare the hemodynamic values measured at different time points, and p-values were corrected with Bonferroni adjustment. Comparison of the hemodynamic measurements in the two groups for the selected time points was performed using the Mann-Whitney U test. A p-value smaller than 0.05 was accepted as statistically significant. 


\section{Results}

A total of 80 women (mean age 26.9 4 .1years) undergoing CS were enrolled in this study. Forty of the participants underwent elective CS, and the other 40 participants underwent emergency CS. The two groups were similar concerning age, body mass index, gravidity, and parity, history of previous CS, ASA class, and anesthesia time (Table 1). The

Table 1. Demographic characteristics and perioperative fluid and vasopressor requirement of the study population

\begin{tabular}{lccc}
\hline & $\begin{array}{c}\text { Emergency CS } \\
\mathbf{n = 4 0}\end{array}$ & $\begin{array}{c}\text { Elective CS } \\
\mathbf{n = 4 0}\end{array}$ & $\mathbf{p}$ \\
\hline Age, years & $27.5 \pm 3.9$ & $26.6 \pm 4.2$ & 0.240 \\
BMI, $\mathrm{kg} / \mathrm{m}^{2}$ & $29.9 \pm 5.1$ & $31.2 \pm 4.2$ & 0.216 \\
Gravidity, $\mathrm{n}$ & $2.3 \pm 1.1$ & $2.8 \pm 1.3$ & 0.095 \\
Parity, $\mathrm{n}$ & $2.1 \pm 1.0$ & $2.5 \pm 1.0$ & 0.165 \\
Previous CS, $\mathrm{n}$ & 12 & 13 & 0.809 \\
ASA class, $\mathrm{n}$ & & & \\
$\quad$ I & 35 & 36 & 0.723 \\
$\quad$ II & 5 & 4 & \\
Anesthesia time, min & $48.8 \pm 5.5$ & $49.4 \pm 5.9$ & 0.669 \\
Iv. fluid, ml & $650 \pm 280$ & $1430 \pm 460$ & $<0.001$ \\
Iv. Ephedrine requirement, $\mathrm{n}$ & $21(52.5 \%)$ & $5(12.5 \%)$ & $<0.001$ \\
\hline
\end{tabular}

amount of iv. fluid administered before surgery was significantly lower in the emergency CS group compared to the elective CS group $650 \pm 280 \mathrm{ml}$ vs. $1430 \pm 460 \mathrm{ml}, \mathrm{p}<0.01$ ). Moreover, intraoperative ephedrine requirement was also significantly higher in the emergency CS group. Table 2 shows the change in the hemodynamic parameters and oxygen saturation in the two groups throughout the anesthesia procedure. A significant drop occurred in systolic, diastolic, and mean arterial pressure early at the $5^{\text {th }}$ minute of anesthesia in patients undergoing emergency CS. In addition, the heart rate significantly increased as a response to the reduction in the arterial pressure. Systolic arterial pressure $(90.7 \pm 20.6 \mathrm{~min}$ vs. $110.2 \pm 11.2, \mathrm{p}<0.001)$ and mean arterial pressure $(68.1 \pm 16.7 \mathrm{~min}$ vs. $75.5 \pm 15.0, \mathrm{p}=0.044)$ of the emergency CS group were significantly lower than those of the elective CS patients beginning from the $15^{\text {th }}$ minute of the anesthesia. However, no significant change was observed in the elective CS group concerning the systolic, diastolic, and mean arterial pressure and heart rate. There were no significant changes in oxygen saturation in both groups.

Table 2. Change in systolic, diastolic and mean arterial pressure, heart rate and oxygen saturation throughout the anesthesia

\begin{tabular}{|c|c|c|c|c|c|c|c|}
\hline & Preinduction & $5^{\text {th }}$ minute & $15^{\text {th }}$ minute & $30^{\text {th }}$ minute & $40^{\text {th }}$ minute & Post-anesthesia & p* \\
\hline \multicolumn{8}{|l|}{ SBP } \\
\hline Elective & $114.6 \pm 9.4$ & $114.2 \pm 12.2$ & $110.2 \pm 11.2$ & $110.3 \pm 11.0$ & $112.3 \pm 11.5$ & $114.1 \pm 11.5$ & 0.065 \\
\hline Emergency & $118.5 \pm 13.3$ & $106.3 \pm 23.1^{a}$ & $90.7 \pm 20.6^{\beta}$ & $95.4 \pm 18.0^{\alpha}, \beta$ & $103.7 \pm 15.9^{a}$ & $108.7 \pm 12.0^{a}$ & $<0.0001$ \\
\hline$p$-value $e^{* *}$ & 0.101 & 0.060 & $<0.001$ & $<0.001$ & 0.007 & 0.045 & \\
\hline \multicolumn{8}{|l|}{ DBP } \\
\hline Elective & $65.3 \pm 8.6$ & $64.62 \pm 9.3$ & $61.6 \pm 10.8$ & $61.8 \pm 9.1$ & $62.7 \pm 8.6$ & $63.7 \pm 8.9$ & 0.075 \\
\hline Emergency & $75.5 \pm 14.3$ & $64.9 \pm 15.9^{a}$ & $55.2 \pm 14.8^{\beta}$ & $57.4 \pm 11.8^{a, \beta}$ & $59.7 \pm 9.6^{a, \beta}$ & $62.5 \pm 9.0^{\alpha, \beta}$ & $<0.0001$ \\
\hline$p$-value $e^{* *}$ & 0.420 & 0.812 & 0.098 & 0.062 & 0.139 & 0.553 & \\
\hline \multicolumn{8}{|l|}{ MAP } \\
\hline Elective & $78.4 \pm 11.5$ & $78.1 \pm 13.3$ & $75.5 \pm 15.0$ & $76.4 \pm 11.7$ & $76.6 \pm 9.6$ & $78.3 \pm 9.2$ & 0.151 \\
\hline Emergency & $85.7 \pm 14.3$ & $76.6 \pm 16.9^{a}$ & $68.1 \pm 16.7$ & $69.5 \pm 12.4^{\alpha}$ & $73.9 \pm 10.2^{a}$ & $76.7 \pm 8.9^{a}$ & $<0.0001$ \\
\hline$p$-value $e^{* *}$ & 0.534 & 0.198 & 0.044 & 0.03 & 0.012 & 0.116 & \\
\hline \multicolumn{8}{|l|}{$\mathrm{HR}$} \\
\hline Elective & $89.4 \pm 13.9$ & $90.4 \pm 14.3$ & $91.2 \pm 13.8$ & $89.9 \pm 13.3$ & $89.2 \pm 13.1$ & $89.8 \pm 11.3$ & 0.493 \\
\hline Emergency & $90.9 \pm 16.3$ & $94.1 \pm 16.0^{a}$ & $95.9 \pm 14.3^{a}$ & $93.0 \pm 13.4$ & $90.1 \pm 14.6$ & $90.6 \pm 12.1$ & 0.018 \\
\hline$p$-value ${ }^{* *}$ & 0.350 & 0.020 & 0.002 & 0.042 & 0.533 & 0.531 & \\
\hline \multicolumn{8}{|l|}{ Sat } \\
\hline Elective & $98.3 \pm 1.9$ & $98.6 \pm 1.8$ & $98.7 \pm 1.5$ & $98.7 \pm 1.5$ & $99.0 \pm 0.6$ & $99.0 \pm 0.6$ & 0.248 \\
\hline Emergency & $98.2 \pm 1.9^{a}$ & $98.5 \pm 1.8^{a}$ & $98.7 \pm 1.5^{a}$ & $98.7 \pm 1.5^{a}$ & $99.0 \pm 0.6^{a}$ & $99.0 \pm 0.6^{a}$ & 0.236 \\
\hline$p$-value ${ }^{* *}$ & 0.876 & 0.703 & 0.766 & 0.766 & 0.732 & 0.863 & \\
\hline
\end{tabular}

$a, \beta=$ Presence of one of the letters indicates the lack of statistical significance when compared to another measurement with the same letter; $p$-value ${ }^{*}=$ The $p$ value for in-group comparison of the hemodynamic parameters at different time points (Paired samples $t$-test); $p$-value ${ }^{* *}=$ The $p$ value for group comparisons of each measurement (Student's t-test). 


\section{Discussion}

The present study aimed to compare the elective and the emergency CS with spinal anesthesia about the hemodynamic changes. Our findings show that a significant drop occurs in arterial pressure, and heart rate increases significantly during the emergency CS while no significant hemodynamic changes are noted in the elective CS patients. The amount of the intravenous fluid administered before surgery was significantly lower, whereas the intraoperative vasopressor requirement was significantly higher in the emergency CS group compared to the elective CS group. Proper volume administration and strict hemodynamic monitorisation may be required to prevent hypotension and related fetal and maternal complications in patients undergoing emergency CS.

Reduction in arterial pressure has long been considered as a critical issue in a patient undergoing CS with spinal anesthesia ${ }^{[7]}$. Nausea and vomiting are the most common conditions associated with spinal hypotension in women undergoing $\mathrm{CS}{ }^{[8]}$. However, more severe maternal complications, including loss of consciousness, aspiration, and even cardiovascular collapse, may occur in rare instances. As a consequence of the prolonged maternal hypotension and the resultant reduction in uteroplacental blood flow, fetal hypoxia and acidosis may develop ${ }^{[9]}$. Hemodynamic control during CS under spinal anesthesia is, therefore, critical for the well-being of both the mother and the fetus.

Cesarean delivery on maternal request (CDMR) is defined as the elective cesarean in the absence of any medical or obstetric contraindication for attempting a vaginal delivery. CDMR is considered as the most common reason for the increasing incidence of cesarean sections ${ }^{[10,11]}$. Spinal anesthesia has been shown to be associated with hypotension in more than $80 \%$ of the parturient if not preemptively managed ${ }^{[12]}$. The decrease in the systemic vascular resistance throughout the pregnancy makes women more vulnerable to the hypotensive effects of the spinal anesthesia. The elective setting provides sufficient time for healthcare professionals to address the volume status and correct preexisting volume depletion. Volume replacement is usually performed even in women without profound volume depletion to overcome the potential hypotension which may occur during the spinal anesthesia ${ }^{[13-15]}$. However, adequate volume replacement cannot be achieved in the majority of the patients requiring emergency $\mathrm{CS}$ and these women are prone to the hypotensive effects of the anesthesia, which is more critical for the women with underlying volume depletion.
Our findings show that compared to the elective CS, emergency CS is associated with a significant reduction in arterial pressure and women undergoing emergency CS more frequently require vasopressor drugs. A possible explanation for this result may be the lack of adequate volume replacement in the emergency CS patients. The significant difference in the amount of the intravenous fluids administered before the CS in our series supports this consideration.

With this in mind, we suggest that patients undergoing emergency CS with spinal anesthesia should be evaluated carefully concerning the volume status and hemodynamic parameters to prevent the development of a dropin arterial pressure during the operation. Developing a multidisciplinary approach to the obstetric patients with the emergency department staff may enable rapid evaluation of volume status and early volume replacement before CS. In addition, intraoperative measures, such as aggressive volume replacement, administration of vasopressor agents, and leg elevation during the emergency, may prevent the development of hypotension during the emergency CS $[9,16]$.

This study has some limitations. First, this study is a retrospective analysis, and the sample size is relatively small. Second, as a handicap of the retrospective data collection, we could not provide detailed information regarding the maternal and fetal of the elective and emergency CS groups. These results, therefore, need to be interpreted with caution.

\section{Conclusion}

In contrast to the women undergoing elective CS, a significant drop occurs in systolic, diastolic and mean arterial blood pressure following the implementation of the spinal anesthesia in patients undergoing emergency CS. Rapid evaluation of the volume status and intravenous volume replacement may prevent the reduction of the arterial pressure in patients undergoing emergency CS. Aggressive volume replacement and administration of vasopressor agents should be considered in spinal hypotension as early as possible to avoid the development of maternal and fetal complications.

Ethics Committee Approval: This study was approved by the Institutional Ethical Committee and was performed in accordance with the recent version of the Helsinki Declaration (27.08.2019/46059653).

Peer-review: Externally peer-reviewed.

Conflict of Interest: None declared.

Financial Disclosure: The authors declared that this study received no financial support. 


\section{References}

1. Yang XJ, Sun SS. Comparison of maternal and fetal complications in elective and emergency cesarean section: a systematic review and meta-analysis. Arch Gynecol Obstet 2017;296:503-12. [CrossRef]

2. Mylonas I, Friese K. Indications for and Risks of Elective Cesarean Section. Dtsch Arztebl Int 2015;112:489-95. [CrossRef]

3. Arikan I, Barut A, Harma M, Harma IM, Gezer S, Ulubasoglu $H$. Cesarean section with relative indications versus spontaneous vaginal delivery: short-term outcomes of maternofetal health. Clin Exp Obstet Gynecol 2012;39:288-92.

4. Lurie S, Shalev A, Sadan O, Golan A.; The changing indications and rates of cesarean section in one academic center over a 16-year period (1997-2012). Taiwan J Obstet Gynecol 2016;55:499-502. [CrossRef]

5. Nagy S. Changing trends and indications for cesarean section in the last few decades. Orv Hetil 2014;155:1140-6. [CrossRef]

6. Gosset M, Ilenko A, Bouyou J, Renevier B. Emergency caesarean section. J Visc Surg 2017;154:47-50. [CrossRef]

7. Toyama S. Hypotension during spinal anaesthesia for caesarean section. Anaesthesia 2015;70:1208-9. [CrossRef]

8. Pellegrini JE. Treatment and Prevention of Spinal-Induced Hypotension in the Cesarean Section Patient: What Does the Evidence Say? Annu Rev Nurs Res 2017;35:159-78. [CrossRef]

9. Heesen M, Klimek M, Hoeks SE, Rossaint R. Prevention of Spinal Anesthesia-Induced Hypotension During Cesarean
Delivery by 5-Hydroxytryptamine-3 Receptor Antagonists: A Systematic Review and Meta-analysis and Meta-regression. Anesth Analg 2016;123:977-88. [CrossRef]

10. Belizan JM, Althabe F, Cafferata ML. Health consequences of the increasing caesarean section rates. Epidemiology 2007;18:485-6. [CrossRef]

11. Villar J, Carroli G, Zavaleta N, Donner A, Wojdyla D, Faundes $A$, et al. Maternal and neonatal individual risks and benefits associated with caesarean delivery: multicentre prospective study. Bmj 2007;335:1025. [CrossRef]

12. Saravanan S, Kocarev M, Wilson RC, Watkins E, Columb MO, Lyons $\mathrm{G}$. Equivalent dose of ephedrine and phenylephrine in the prevention of post-spinal hypotension in Caesarean section. Br J Anaesth 2006;96:95-9. [CrossRef]

13. Bishop DG, Cairns C, Grobbelaar M, Rodseth RN. Obstetric spinal hypotension: Preoperative risk factors and the development of a preliminary risk score - the PRAM score. S Afr Med J 2017; 107:1127-31. [CrossRef]

14. Lapage KG, Wouters PF. The patient with hypertension undergoing surgery. Curr Opin Anaesthesiol 2016;29:397-402.

15. Nicholas JA. Preoperative optimization and risk assessment. Clin Geriatr Med 2014;30:207-18. [CrossRef]

16. Hasanin A, Aiyad A, Elsakka A, Kamel A, Fouad R, Osman M, et al. Leg elevation decreases the incidence of post-spinal hypotension in cesarean section: a randomized controlled trial. BMC Anesthesiol 2017;17:60. [CrossRef] 